\title{
The Suprachiasmatic Nucleus Entrains, But Does Not Sustain, Circadian Rhythmicity in the Olfactory Bulb
}

\author{
Daniel Granados-Fuentes, Laura M. Prolo, Ute Abraham, and Erik D. Herzog \\ Department of Biology, Washington University, St. Louis, Missouri 63130-4899
}

The suprachiasmatic nucleus (SCN) of the hypothalamus has been termed the master circadian pacemaker of mammals. Recent discoveries of damped circadian oscillators in other tissues have led to the hypothesis that the SCN synchronizes and sustains daily rhythms in these tissues. We studied the effects of constant lighting (LL) and of SCN lesions on behavioral rhythmicity and Period 1 (Per1) gene activity in the SCN and olfactory bulb (OB). We found that LL had similar effects on cyclic locomotor and feeding behaviors and Per1 expression in the SCN but had no effect on rhythmic Period 1 expression in the OB. LL lengthened the period of locomotor and SCN rhythms by $\sim 1.6 \mathrm{hr}$. After 2 weeks in LL, nearly $35 \%$ of rats lost behavioral rhythmicity. Of these, $90 \%$ showed no rhythm in Per1-driven expression in their SCN. Returning the animals to constant darkness rapidly restored their daily cycles of running wheel activity and gene expression in the SCN. In contrast, the $\mathrm{OB}$ remained rhythmic with no significant change in period, even when cultured from animals that had been behaviorally arrhythmic for 1 month. Similarly, we found that lesions of the SCN abolished circadian rhythms in behavior but not in the OB. Together, these results suggest that LL causes the SCN to lose circadian rhythmicity and its ability to coordinate daily locomotor and feeding rhythms. The SCN, however, is not required to sustain all rhythms because the $\mathrm{OB}$ continues to oscillate in vivo when the SCN is arrhythmic or ablated.

Key words: hypothalamus; locomotion; oscillator; pacemaker; rhythm; Period 1 gene

\section{Introduction}

The suprachiasmatic nucleus (SCN) of the hypothalamus has been termed the master circadian pacemaker in mammals (Reppert and Weaver, 2001). Recently, however, other tissues have been found to also show endogenous circadian rhythmicity in transcriptional activity (Balsalobre et al., 2000b; Yamazaki et al., 2000; Balsalobre, 2002; Tosini and Fukuhara, 2002) or melatonin release (Tosini and Menaker, 1996). This seems at odds with the findings that SCN lesions (SCNX) abolish circadian rhythms in behavior, endocrine function (cf. Lehman et al., 1991), electrical activity in various brain areas (Inouye and Kawamura, 1979; Kubota et al., 1981), and gene transcription in rats (Sakamoto et al., 1998; Akhtar et al., 2002). These results have led to the hypothesis that the SCN synchronizes and sustains daily rhythms in these tissues (Balsalobre et al., 1998; Yamazaki et al., 2000). A hierarchical model has been proposed in which the SCN may entrain rhythmicity in a variety of tissues (cf. Herzog and Tosini, 2001). Little is known about the interactions within this multioscillator system. The connections appear to be relatively weak because rapid changes in the light or feeding schedule can alter the normal phase relationships between the SCN and these other oscillators for many days (Balsalobre et al., 2000a; Damiola et al.,

\footnotetext{
Received Aug. 29, 2003; revised 0ct. 30, 2003; accepted 0ct. 31, 2003

This work was supported by National Institutes of Health Grant MH63104 and the MCDonnell Center for Higher Brain Function. We are indebted to Dr. Hajime Tei (University of Tokyo, Tokyo, Japan) for generously providing Per1-luc rats. We thank Sara Aton, Meera Saxena, Paul Stein, and Paul Taghert for comments on this manuscript.

Correspondence should be addressed to Erik Herzog, Department of Biology, Box 1137, Washington University, St. Louis, M0 63130-4899. E-mail: herzog@biology.wustl.edu.

DOI:10.1523/JNEUROSCI.4002-03.2004

Copyright $\odot 2004$ Society for Neuroscience $\quad$ 0270-6474/04/240615-05\$15.00/0
}

2000; Yamazaki et al., 2000; Le Minh et al., 2001; Stokkan et al., 2001; Ohta et al., 2002).

This study focuses on the effects of constant light (LL) and SCNX on the coordination of circadian timing in the SCN and the olfactory bulb (OB). Prolonged illumination has been shown to abolish circadian rhythms in a variety of organisms, including mammals (Winfree, 1974; Depres-Brummer et al., 1995; Power et al., 1995; Honma et al., 1996; Cambras et al., 1997; Ikeda et al., 2000 ). We chose the OB primarily because of its robust rhythmicity in vitro (Abe et al., 2002) and indirect evidence implicating it in circadian timekeeping. For example, removal of the bulbs lengthens the free-running periods of hamsters and mice (Possidente et al., 1990; Pieper and Lobocki, 1991) and slows photic reentrainment in male Octogon degus (Goel and Lee, 1995, 1997; Lee and Labyak, 1997; Goel et al., 1998). Polysynaptic projections from the OB to the SCN have been reported previously (Krout et al., 2002), and olfactory stimulation can enhance light-induced phase shifts of locomotor activity and Fos expression in the SCN (Amir et al., 1999b). Finally, there is evidence for circadian modulation of odor-evoked activity in the bulb (Amir et al., 1999a).

We measured the effects of LL and SCN lesions on behavioral and Period 1 (Per1) gene activity in the SCN and OB. Perl is one of approximately eight genes implicated in circadian rhythm generation (Allada et al., 2001). We found that continuous bright light or SCN lesions can abolish daily rhythms in behaviors and in gene expression of the $\mathrm{SCN}$ but not of the $\mathrm{OB}$, indicating that the $\mathrm{SCN}$ is not required for all $24 \mathrm{hr}$ timekeeping functions in mammals.

\section{Materials and Methods}

Male, homozygous Per1-luc rats were individually housed at 3 weeks of age in a cage outfitted with a running wheel. Cages were placed in venti- 
lated chambers illuminated by fluorescent bulbs $\left(3.9 \times 10^{17}\right.$ to $6.9 \times 10^{18}$ photons $\cdot \mathrm{sec}^{-1} \cdot \mathrm{m}^{2}$ at the bottom of the cages; F30T12-SP41-RS, General Electric). Wheelrunning activity was recorded in 6 min bins (Clocklab; Actimetrics, Evanston, IL) until the animals were killed. Food consumption was measured every $3 \mathrm{hr}$ by weighing food in the cage lid. All procedures were approved by the Animal Care and Use Committee and conformed to National Institutes of Health guidelines.

Real time Per1-luc measurement. Per1 gene activity was assessed by measuring bioluminescence from cultured tissues harvested from Per1-luc rats using methods similar to previous reports (Abe et al., 2002). After 2 weeks in a 12 hr light/dark (LD) cycle, control animals $(n=$ 5) were killed, and experimental animals were switched to constant light conditions (LL). Experimental animals were killed after 29-32 d $(n=12), 34-48 \mathrm{~d}(n=11)$, or $50-66 \mathrm{~d}(n=7)$ in LL or transferred back to constant darkness (DD) for $5 \mathrm{~d}$, after which their SCN and OB were explanted and recorded $(n=11$ rats for behavior and 3 for physiology). The time of lights on in the animal colony was defined as Zeitgeber time 0 (ZT 0) and lights off as ZT 12. The time of projected locomotor activity onset in constant conditions (DD or LL) was defined as circadian time 12 (CT 12). Surgery was performed $\sim 60$ min before dusk or when the animals were in constant conditions, before projected onset of daily locomotor activity. For animals that were arrhythmic, we extrapolated from the last time they were clearly rhythmic to define the time of projected onset of activity (so that $\mathrm{CT}$ and time since surgery are synonymous in all figures).

Rats were anesthetized with $\mathrm{CO}_{2}$ and decapitated, and their brains were rapidly removed and placed in cold HBSS (14060-057; Invitrogen, Gaithersburg, MD). Coronal sections (300- $\mu \mathrm{m}$-thick) were made with a vibratome.

Brain regions were identified under a dissecting microscope (Paxinos and Watson, 1998). The SCNs were isolated as a square of tissue, $\sim 1.5$ $\mathrm{mm}$ across with a pair of scalpels; the $\mathrm{OB}$ was cut $5.7-6.7 \mathrm{~mm}$ anterior to bregma. Tissues were placed on Millicell membranes (PICM0RG-50; Millipore, Bedford, MA) with $1.2 \mathrm{ml}$ of DMEM (13000-021; Invitrogen) supplemented with $10 \mathrm{~mm}$ HEPES, $100 \mathrm{U} / \mathrm{ml}$ penicillin, $100 \mu \mathrm{g} / \mathrm{ml}$ streptomycin, and $0.1 \mathrm{~mm}$ beetle luciferin (E1601; Promega, Madison, WI). Each explant was sealed in a $35 \mathrm{~mm}$ Petri dish with a coverslip and vacuum grease and maintained at $36^{\circ} \mathrm{C}$ in darkness, and their bioluminescence was continuously monitored with a photomultiplier tube (HC135-11MOD; Hamamatsu, Shizouka, Japan) for 3-8 d.

SCN lesions. Locomotor activity was recorded for $5 \mathrm{~d}$ from rats in a 12 $\mathrm{hr}$ LD cycle. Rats were then anesthetized with ketamine $(75 \mathrm{mg} / \mathrm{kg})$ and medetomidine $(0.5 \mathrm{mg} / \mathrm{kg}$, i.p.) and placed in a stereotaxic apparatus (David Kopf Instruments, Tujunga, CA). Bilateral SCNX $(n=5)$ were made by passing an anodal direct current $(1.25 \mathrm{~mA}$, for $1 \mathrm{~min})$ through a tungsten electrode (563410; A-M systems, Carlsborg, WA) placed 0.4 $\mathrm{mm}$ posterior to, $\pm 0.1 \mathrm{~mm}$ lateral to, and $8.7 \mathrm{~mm}$ below bregma (Paxinos and Watson, 1998). Control animals $(n=4)$ were treated identically but without passing current through the electrode. Wheel-running activity was then monitored in DD until animals were killed 2 weeks $(n=2$ sham and $n=3$ SCNX) or 3 weeks ( $n=2$ sham and $n=2$ SCNX) after the lesion. The $\mathrm{OB}$ was harvested, and bioluminescence was recorded as described above. In two SCNX rats, the area around and including the lesion was also cultured to evaluate the potential for residual rhythmicity in the remaining tissue. SCN lesions were confirmed in the remaining three rats from Nissl-stained cryosections.

Data analysis. Bioluminescence data were detrended by subtracting the $24 \mathrm{hr}$ running average from the raw data, and the phase of the first peak was determined as described previously (Abe et al., 2002). Period (range of 18-32 hr) and arrhythmicity of bioluminescence, feeding, and locomotor activity were calculated at the $95 \%$ confidence level using $\chi^{2}$ periodogram and fast Fourier transform-nonlinear least-squares fit analysis (Sokolove and Bushell, 1978; Herzog et al., 1997). The two methods identified the same records as arrhythmic. Differences in period were analyzed by a one-way ANOVA, followed by Tukey's post hoc test. Periods are expressed as mean \pm SEM. We used the Rayleigh test to determine whether the distribution of phases within the different groups were significantly different from a uniform distribution at the $95 \%$ confidence level (Batschelet, 1981).

\section{Results}

\section{Constant illumination abolishes circadian rhythms in feeding and locomotion}

We measured food consumption and running wheel activity from transgenic rats in which the mouse Period 1 promoter drives luciferase (Per1-luc) transcription (Yamazaki et al., 2000). All rats $(n=30)$ synchronized to a $12 \mathrm{hr}$ LD cycle and then lengthened their running wheel rhythms during the first 2 weeks in LL (Fig. 1). After $13 \mathrm{~d}$ in LL, 20 rats had a period of $25.6 \pm 0.06 \mathrm{hr}$, and 10 


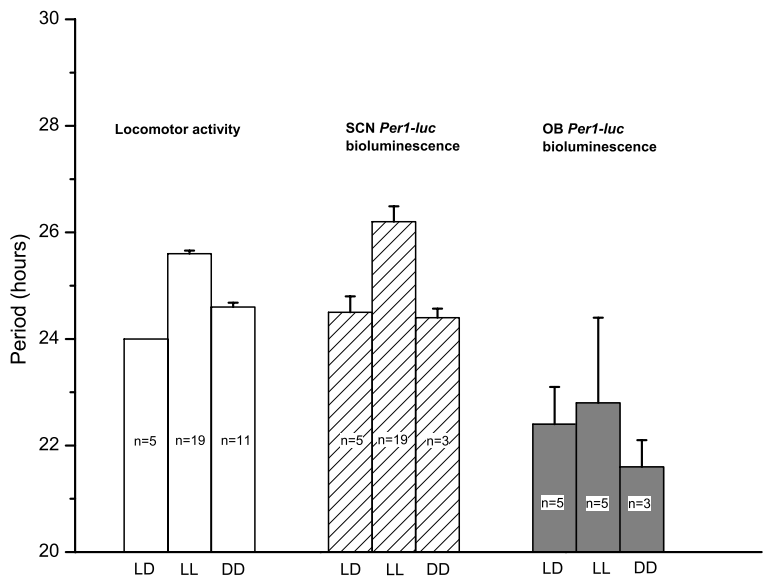

Figure 2. The period of the $S C N$, but not the $O B$, depended on lighting conditions and change with the behavioral period. LL lengthened the period of locomotor activity $(25.6 \pm 0.1 \mathrm{hr}$ mean \pm SEM) and of SCN bioluminescence rhythms ( $26.2 \pm 0.3 \mathrm{hr}$ ) by $\sim 1.6 \mathrm{hr}$ from LD entrained conditions. When animals were switched from $L L$ to DD, locomotor activity (24.6 \pm $0.06 \mathrm{hr}$ ) and Per 1-luc ( $24.4 \pm 0.17 \mathrm{hr}$ ) periods significantly shortened. In contrast, the period of the cultured $\mathrm{OB}$ did not significantly change after any of the illumination conditions ( $22.4 \pm 0.7$ hr for $L D ; 22.8 \pm 1.6 \mathrm{hr}$ for $\mathrm{LL} ; 21.6 \pm 0.5 \mathrm{hr}$ for DD after $\mathrm{LL}$; comparison of LD vs $\mathrm{LL}$ vs DD locomotor activity, $F_{(2,32)}=132, p<0.001$; SCN Per1-luc activity, $F_{(2,24)}=7.07, p<0.005$; OB Per1-luc activity, $F_{(2,12)}=0.15, p=0.86$; one-way ANOVA and Tukey's test).

rats became arrhythmic. Food consumption, although statistically rhythmic in $78 \%$ of animals in LD, became arrhythmic in all animals tested after 2 weeks in LL $(n=9)$.

\section{The OB oscillate, whereas the SCN do not, in arrhythmic animals}

We correlated behavioral patterns with Per1-luc expression in SCN and OB explants from animals killed after 5,6 , or 8 weeks in LL. The SCN of $90 \%$ of the arrhythmic animals showed no circadian rhythm ( $n=9$ of 10 ) (Fig. 1$)$, whereas $95 \%$ of the SCN from behaviorally rhythmic rats were rhythmic $(n=19$ of 20$)$. This strong correlation held, even after $50 \mathrm{~d}$ in LL when all animals that remained rhythmic had rhythmic SCN in vitro $(n=3)$ and arrhythmic animals had arrhythmic SCN $(n=4)$. In contrast, all animals tested, behaviorally rhythmic or not, had rhythmic OB $(n=13)$ (Fig. 2). Thus, constant light affects Perl cycling in the SCN but not in the OB.

Prolonged exposure to light could compromise the health of SCN cells, stop their pacemaking, or desynchronize them from one another. To assess whether arrhythmic animals were capable of generating circadian rhythmicity, we switched them from LL to DD. Within $2 \mathrm{~d}$, these rats expressed an average free-running period of $24.6 \pm 0.1 \mathrm{hr}(n=11)$ (Fig. 2), in agreement with previous reports (Depres-Brummer et al., 1995). Restored behavioral rhythmicity correlated with restored Per1-luc rhythmicity in the SCN (periods were $24.4 \pm 0.2 \mathrm{hr} ; n=3$ ) and continued rhythmicity in the $\mathrm{OB}(21.6 \pm 0.5 \mathrm{hr} ; n=3)$. Furthermore, arrhythmic SCN explants $(n=5)$ reliably resumed rhythmicity after a 30 min pulse of $0.2 \mathrm{~mm}$ forskolin, an activator of adenylate cyclase. These results confirm that arrhythmic SCN were not in poor health and could rapidly resume rhythmicity when presented with an adequate stimulus. Thus, LL either stops the pacemaker, as suggested in Drosophila (Marrus et al., 1996), or disrupts the coordinate timing of the pacemakers within the SCN, as suggested in hamsters (de la Iglesia et al., 2000).

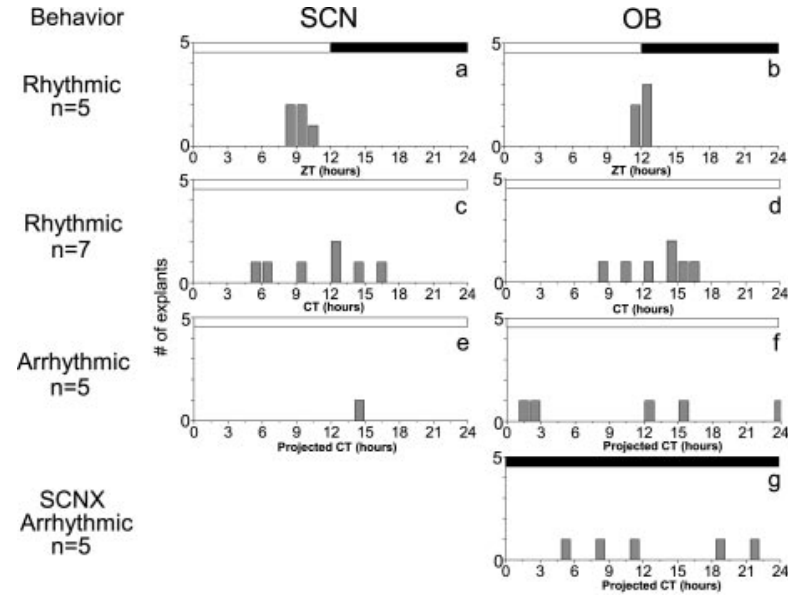

Figure 3. The time of peak Per1-luc expression in $\mathrm{SCN}$ and $\mathrm{OB}$ cultures was tightly regulated in LD but disrupted by LL. The time of the first peak of each explant in bioluminescence provided a phase marker for in vivo rhythmicity relative to the time of light onset in the animal colony (ZT 0 ), activity onset (CT 12), or projected activity onset had they remained behaviorally rhythmic (CT 12). a, b, SCN cultures from animals kept in LD cycles peaked $\sim 9.5 \mathrm{hr}$ after the time of projected dawn (ZT $9.5 \mathrm{hr} ; p=0.001 ; r=0.98$; Rayleigh test), whereas $0 \mathrm{~B}$ cultures peaked $\sim 2.5 \mathrm{hr}$ later (ZT $12.0 \mathrm{hr} ; p=0.001 ; r=0.98) . c, d$, SCN cultures from rhythmic animals kept in LL did not show significant phase clustering ( $p=0.1 ; r=0.57)$. OB cultures from the same animals, however, peaked at $\mathrm{CT} 10.7 \mathrm{hr}(p=0.006 ; r=0.8)$.e, $f, 0$ the subset of animals that became behaviorally arrhythmic in $\mathrm{LL}$, only one had a rhythmic SCN in vitro, although all had rhythmic $\mathrm{OBs}$ that peaked at variable times $(p=0.7 ; r=0.3) . g$, Similarly, the $\mathrm{OB}$ of animals rendered behaviorally arrhythmic by $S C N$ ablation also showed a broadened distribution at peak times ( $p=0.47 ; r=0.4$ ). The times of the previous light (open bars) and dark (filled bars) schedule are shown above each plot.

\section{The phase of $\mathrm{OB}$ and SCN oscillations can vary independently in vivo}

Constant illumination gradually disrupted the phasing of SCN and $\mathrm{OB}$ rhythms, indicating a level of independence between the two structures in vivo. We showed previously that the phase of Per1-luc rhythmicity is similar in vivo and in vitro (Abe et al., 2002). SCN explants taken from $L D$ rats peaked an average of 2.5 hr before OB explants (ZT 9.5 $\pm 0.8 \mathrm{hr}$ for SCN explants; ZT $12.0 \pm 0.5 \mathrm{hr}$ for $\mathrm{OB}$ explants; mean \pm angular deviation; $n=5$ each) (Fig. 3a,b) in agreement with published results (Abe et al., 2002). LL broadened the distribution of phases so that, whereas OB cultures peaked at CT $10.7 \pm 2.4 \mathrm{hr}(n=7)$ (Fig. $3 d)$, SCN cultures no longer peaked at a reliable time relative to the time of surgery or locomotor activity $(n=7)$ (Fig. $3 c)$. Thus, LL abolished the phase relationship between the SCN and the OB in behaviorally rhythmic animals (Fig. 4). In arrhythmic animals (Fig. 3f), OB cultures peaked at variable times, indicating that the oscillations in the $\mathrm{OB}$ were not induced by the explant preparation.

\section{The OB continues to oscillate in SCN-lesioned animals}

To directly assess whether the SCN is required for sustained rhythmicity in the OB, we ablated the bilateral SCN. Destruction of the SCN abolished circadian rhythms of wheel-running activity in all rats $(n=5)$ (Fig. $1 p)$, whereas sham-operated animals continued to express circadian rhythmicity with a free-running period of $24.4 \pm 0.1 \mathrm{hr}(n=4)$. The OB from all of these animals remained rhythmic, even 3 weeks after the SCN was lesioned (Fig. $1 q)$, with average periods of $22.6 \pm 1 \mathrm{hr}(\mathrm{SCNX})$ and $23.5 \pm 1.1 \mathrm{hr}$ (sham). Similar to results from animals in LL, bioluminescence peaked at ZT $11.1 \pm 1.9 \mathrm{hr}(p=0.02 ; r=0.9 ; n=4$; data not shown) in the $\mathrm{OB}$ of control rats but peaked at variable times in 


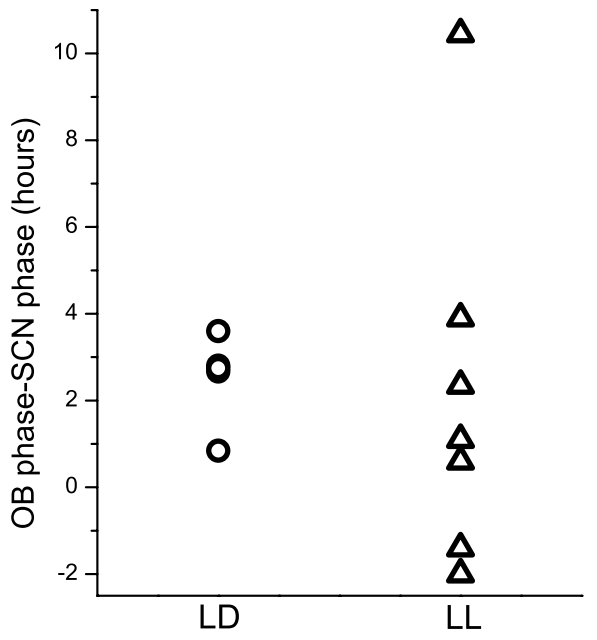

Figure 4. The normal phase relationship between the $\mathrm{SCN}$ and $\mathrm{OB}$ is disrupted by constant light. The phase relationship between $\mathrm{SCN}$ and $\mathrm{OB}$ explants was calculated by subtracting the time of the first peak in Per1-luc expression of the SCN from that of the OB. Positive values thus indicate when the rhythm in the $\mathrm{SCN}$ preceded that in the $\mathrm{OB}$ from the same animal. Constant light abolished the reliable lag from $\mathrm{SCN}$ to $0 B$ seen in $\mathrm{LD}$. Lag was statistically grouped at $2.5 \mathrm{hr}$ ( $p=0.01 ; r=0.97)$ for $\mathrm{LD}$, whereas the $\mathrm{LL}$ group was not grouped ( $p=0.06 ; r=0.6)$.

SCNX animals $(n=5)$ (Fig. $3 g$ ). The remaining tissue in the vicinity of the lesion failed to express Per1-driven rhythms $(n=$ 2 ), suggesting that the rhythmicity observed in the OB was not sustained by residual rhythmicity in the hypothalamus.

\section{Discussion}

We found previously that the $\mathrm{OB}$ and several other brain areas can sustain rhythmicity for a few days after explantation from rhythmic animals (Abe et al., 2002). The present results show that the $\mathrm{OB}$ is still rhythmic when explanted from animals that have been behaviorally arrhythmic for $>1$ month. Because lengthening or loss of periodicity in the SCN or ablation of the SCN had no apparent effect on the $\mathrm{OB}$, we conclude that the $\mathrm{OB}$ can oscillate independently and does not require signals from the SCN for sustained rhythmicity. This near $22 \mathrm{hr}$ oscillation is intrinsic to the mitral, not granule, cell layer, can persist in vitro for as long as $16 \mathrm{~d}$ (longest time tested), and is normally synchronized to the onset of locomotor activity (Granados-Fuentes et al., 2004). It is not known whether the persistence of rhythmicity in the $\mathrm{OB}$ depends on signals from areas outside the SCN.

The dramatic effects of LL on Perl gene expression in the SCN continue to support the conventional model that the SCN is the master circadian pacemaker driving daily rhythms in locomotor and feeding activity. In addition, the SCN likely regulates the phase of the OB rhythms in a light cycle because the phase of the OB reliably lags that of the SCN by $2.5 \mathrm{hr}$. We found, however, that LL disrupts the normal phase relationship between the SCN and the OB. This "internal desynchrony" is reminiscent of early experiments on humans that found that LL selectively alters the timing of different physiological rhythms and that were considered to reflect decoupling of multiple circadian tissues (Aschoff and Wever, 1976). Rapid changes in feeding or lighting schedules, associated with jet lag, also dissociate the normal phase relationships between circadian tissues (Balsalobre et al., 2000a; Stokkan et al., 2001; Abe et al., 2002). Together, these results indicate that the consequences of arrhythmicity in the SCN include behavioral arrhythmicity and free-running rhythms in the OB. Tissues, such the $\mathrm{OB}$, appear to entrain to light cycles via relatively weak, rhythmic signals from the SCN.
The rhythms in the OB were unlikely to have been induced when the tissue was placed in vitro. If this were the case, we would expect the phase of OB rhythmicity to be determined by the time of surgery (which was always at the time predicted for locomotor activity onset). $\mathrm{OB}$ cultures, which reliably peak at approximately the time of locomotor activity onset when taken from animals in a light cycle, tend to peak $\sim 1.3 \mathrm{hr}$ earlier when taken from rhythmic animals in LL and show a random distribution of phases when taken from animals rendered arrhythmic by either LL or SCNX. In addition, the same surgery did not induce rhythmicity in the SCN when a forskolin pulse could. Thus, the first peak of the $\mathrm{OB}$ in vitro likely reflects the phase of Per1 rhythmicity in vivo, as has been shown previously for the SCN and pineal (Abe et al., 2002; Wilsbacher et al., 2002).

Remarkably, the effects of LL on the SCN in vivo appear to persist in vitro, as seen here in Perl gene expression and as reported for multiunit firing rates (Mason, 1991). Prolonged exposure to light may stop circadian timekeeping, as suggested in Drosophila in which LL accelerates the degradation of Timeless and Period proteins (Marrus et al., 1996). Alternatively, LL may desynchronize multiple oscillators, as has been implicated in the splitting of locomotor behavior (cf. de la Iglesia et al., 2000). The present results do not eliminate either of these possibilities because LL did not appear to induce changes in the level of Per1 expression or the number of peaks per day in the SCN. Future recordings of firing rate from individual SCN neurons could distinguish between the potential mechanisms by which LL abolishes rhythms in the SCN tissue.

\section{References}

Abe M, Herzog ED, Yamazaki S, Straume M, Tei H, Sakaki Y, Menaker M, Block GD (2002) Circadian rhythms in isolated brain regions. J Neurosci 22:350-356.

Akhtar RA, Reddy AB, Maywood ES, Clayton JD, King VM, Smith AG, Gant TW, Hastings MH, Kyriacou CP (2002) Circadian cycling of the mouse liver transcriptome, as revealed by cDNA microarray, is driven by the suprachiasmatic nucleus. Curr Biol 12:540-550.

Allada R, Emery P, Takahashi JS, Rosbash M (2001) Stopping time: the genetics of fly and mouse circadian clocks. Annu Rev Neurosci 24:1091-1119.

Amir S, Cain S, Sullivan J, Robinson B, Stewart J (1999a) In rats, odorinduced Fos in the olfactory pathways depends on the phase of the circadian clock. Neurosci Lett 272:175-178.

Amir S, Cain S, Sullivan J, Robinson B, Stewart J (1999b) Olfactory stimulation enhances light-induced phase shifts in free- running activity rhythms and Fos expression in the suprachiasmatic nucleus. Neuroscience 92:1165-1170.

Aschoff J, Wever RA (1976) Human circadian rhythms: a multioscillator system. Fed Proc 35:2326-2332.

Balsalobre A (2002) Clock genes in mammalian peripheral tissues. Cell Tissue Res 309:193-199.

Balsalobre A, Damiola F, Schibler U (1998) A serum shock induces circadian gene expression in mammalian tissue culture cells. Cell 93:929-937.

Balsalobre A, Brown SA, Marcacci L, Tronche F, Kellendonk C, Reichardt HM, Schutz G, Schibler U (2000a) Resetting of circadian time in peripheral tissues by glucocorticoid signaling. Science 289:2344-2347.

Balsalobre A, Marcacci L, Schibler U (2000b) Multiple signaling pathways elicit circadian gene expression in cultured Rat-1 fibroblasts. Curr Biol 10:1291-1294.

Batschelet E (1981) Circular statistics in biology. In: Mathematics in biology (Sibson R, Cohen JE, eds), pp 31-54. New York: Academic.

Cambras T, Canal MM, Torres A, Vilaplana J, Diez-Noguera A (1997) Manifestation of circadian rhythm under constant light depends on lighting conditions during lactation. Am J Physiol 272:R1039-R1046.

Damiola F, Le Minh N, Preitner N, Kornmann B, Fleury-Olela F, Schibler U (2000) Restricted feeding uncouples circadian oscillators in peripheral tissues from the central pacemaker in the suprachiasmatic nucleus. Genes Dev 14:2950-2961. 
de la Iglesia HO, Meyer J, Carpino Jr A, Schwartz WJ (2000) Antiphase oscillation of the left and right suprachiasmatic nuclei. Science 290:799-801.

Depres-Brummer P, Levi F, Metzger G, Touitou Y (1995) Light-induced suppression of the rat circadian system. Am J Physiol 268:R1111-R1116.

Goel N, Lee TM (1995) Sex differences and effects of social cues on daily rhythms following phase advances in Octodon degus. Physiol Behav 58:205-213.

Goel N, Lee TM (1997) Olfactory bulbectomy impedes social but not photic reentrainment of circadian rhythms in female Octodon degus. J Biol Rhythms 12:362-370.

Goel N, Lee TM, Pieper DR (1998) Removal of the olfactory bulbs delays photic reentrainment of circadian activity rhythms and modifies the reproductive axis in male Octodon degus. Brain Res 792:229-236.

Granados-Fuentes D, Saxena MT, Prolo LM, Aton SJ, Herzog ED (2004) Olfactory bulb neurons express functional, entrainable circadian rhythms. Eur J Neurosci, in press.

Herzog ED, Tosini G (2001) The mammalian circadian clock shop. Semin Cell Dev Biol 12:295-303.

Herzog ED, Geusz ME, Khalsa SBS, Straume M, Block GD (1997) Circadian rhythms in mouse suprachiasmatic nucleus explants on multimicroelectrode plates. Brain Res 757:285-290.

Honma S, Kanematsu N, Katsuno Y, Honma K-I (1996) Persistence of circadian oscillation while locomotor activity and plasma melatonin levels became aperiodic under prolonged continuous light in the rat. Neurosci Lett 216:49-52.

Ikeda M, Sagara M, Inoue S (2000) Continuous exposure to dim illumination uncouples temporal patterns of sleep, body temperature, locomotion and drinking behavior in the rat. Neurosci Lett 279:185-189.

Inouye ST, Kawamura H (1979) Persistence of circadian rhythmicity in a mammalian hypothalamic "island" containing the suprachiasmatic nucleus. Proc Natl Acad Sci USA 76:5962-5966.

Krout KE, Kawano J, Mettenleiter TC, Loewy AD (2002) CNS inputs to the suprachiasmatic nucleus of the rat. Neuroscience 110:73-92.

Kubota A, Inouye ST, Kawamura H (1981) Reversal of multiunit activity within and outside the suprachiasmatic nucleus in the rat. Neurosci Lett 27:303-308

Le Minh N, Damiola F, Tronche F, Schutz G, Schibler U (2001) Glucocorticoid hormones inhibit food-induced phase-shifting of peripheral circadian oscillators. EMBO J 20:7128-7136.

Lee TM, Labyak SE (1997) Free-running rhythms and light- and dark-pulse phase response curves for diurnal Octodon degus (Rodentia). Am J Physiol 273:R278-R286.

Lehman MN, Silver R, Bittman EL (1991) Anatomy of suprachiasmatic nucleus grafts. In: Suprachiasmatic nucleus: the mind's clock (Klein DC, Moore RY, Reppert SM, eds), pp 349-374. New York: Oxford UP.
Marrus SB, Zeng H, Rosbash M (1996) Effect of constant light and circadian entrainment of perS flies: evidence for light-mediated delay of the negative feedback loop in Drosophila. EMBO J 15:6877-6886.

Mason R (1991) The effects of continuous light exposure on Syrian hamster suprachiasmatic $(\mathrm{SCN})$ neuronal discharge activity in vitro. Neurosci Lett 123:160-163.

Ohta H, Honma S, Abe H, Honma K (2002) Effects of nursing mothers on rPer1 and rPer2 circadian expressions in the neonatal rat suprachiasmatic nuclei vary with developmental stage. Eur J Neurosci 15:1953-1960.

Paxinos G, Watson C (1998) The rat brain in stereotaxic coordinates. New York: Academic.

Pieper DR, Lobocki CA (1991) Olfactory bulbectomy lengthens circadian period of locomotor activity in golden hamsters. Am J Physiol 261:R973-R978.

Possidente B, Lumia AR, McGinnis MY, Teicher MH, deLemos E, Sterner L, Deros L (1990) Olfactory bulb control of circadian activity rhythm in mice. Brain Res 513:325-328.

Power JM, Ringo JM, Dowse HB (1995) The effects of period mutations and light on the activity rhythms of Drosophila melanogaster. J Biol Rhythms 10:267-280.

Reppert SM, Weaver DR (2001) Molecular analysis of mammalian circadian rhythms. Annu Rev Physiol 63:647-676.

Sakamoto K, Nagase T, Fukui H, Horikawa K, Okada T, Tanaka H, Sato K, Miyake Y, Ohara O, Kako K, Ishida N (1998) Multitissue circadian expression of rat period homolog (rPer2) mRNA is governed by the mammalian circadian clock, the suprachiasmatic nucleus in the brain. J Biol Chem 273:27039-27042.

Sokolove PG, Bushell WN (1978) The chi square periodogram: its utility for analysis of circadian rhythms. J Theor Biol 72:131-160.

Stokkan KA, Yamazaki S, Tei H, Sakaki Y, Menaker M (2001) Entrainment of the circadian clock in the liver by feeding. Science 291:490-493.

Tosini G, Fukuhara C (2002) The mammalian retina as a clock. Cell Tissue Res 309:119-126.

Tosini G, Menaker M (1996) Circadian rhythms in cultured mammalian retina. Science 272:419-421.

Wilsbacher LD, Yamazaki S, Herzog ED, Song EJ, Radcliffe LA, Abe M, Block G, Spitznagel E, Menaker M, Takahashi JS (2002) Photic and circadian expression of luciferase in mPeriod1-luc transgenic mice in vivo. Proc Natl Acad Sci USA 99:489-494.

Winfree AT (1974) Suppressing drosophila circadian rhythm with dim light. Science 183:970-972.

Yamazaki S, Numano R, Abe M, Hida A, Takahashi R, Ueda M, Block GD, Sakaki Y, Menaker M, Tei H (2000) Resetting central and peripheral circadian oscillators in transgenic rats. Science 288:682-685. 